\title{
Temperature dependence of electrical conductivity in double-wall and multi-wall carbon nanotube/polyester nanocomposites
}

\author{
Yılmaz Simsek · Lutfi Ozyuzer · A. Tugrul Seyhan • \\ Metin Tanoglu $\cdot$ Karl Schulte
}

Received: 12 September 2006/Accepted: 14 June 2007/Published online: 30 July 2007

(C) Springer Science+Business Media, LLC 2007

\begin{abstract}
The aim of this study is to investigate temperature dependence of electrical conductivity of carbon nanotube (CNT)/polyester nanocomposites from room temperature to $77 \mathrm{~K}$ using four-point probe test method. To produce nanocomposites, various types and amounts of CNTs (0.1, 0.3 and 0.5 wt.\%) were dispersed via 3-roll mill technique within a specially formulized resin blend of thermoset polyesters. CNTs used in the study include multi walled carbon nanotubes (MWCNT) and double-walled carbon nanotubes (DWCNT) with and without amine functional groups $\left(-\mathrm{NH}_{2}\right)$. It was observed that the incorporation of carbon nanotubes into resin blend yields electrically percolating networks and electrical conductivity of the resulting nanocomposites increases with increasing amount of nanotubes. However, nanocomposites containing amino functionalized carbon nanotubes exhibit relatively lower electrical conductivity compared to those with non-functionalized carbon nanotubes. To get better interpretation of the mechanism leading to conductive network via CNTs with and without amine functional groups, the experimental results were fitted to fluctuation-induced tunneling through the barriers between the metallic regions
\end{abstract}

Y. Simsek · L. Ozyuzer

Department of Physics, Izmir Institute of Technology, Izmir, Turkey

A. T. Seyhan · M. Tanoglu $(\bowtie)$

Department of Mechanical Engineering, Izmir Institute of

Technology, Gulbahce Campus, Urla, Izmir 35430, Turkey

e-mail: metintanoglu@iyte.edu.tr

K. Schulte

Polymer Composites, Technische Universität Hamburg-Harburg

(TUHH), Hamburg, Germany model. It was found that the results are in good agreement with prediction of proposed model.

\section{Introduction}

Since their discovery in 1991 by Ijima [1], carbon nanotubes (CNTs) in diameters of a few nanometers have been great study of interest in science. CNTs with their high aspect ratio exhibit extraordinary mechanical, electrical and thermal properties, which make them ideal fillers for advanced composite materials. Two main types of carbon nanotubes are currently accessible; single-walled carbon nanotubes (SWCNT) and multi-walled carbon nanotubes (MWCNT). A special type of the multi-walled tubes is called double-walled carbon nanotubes (DWCNT), which are composed of just two concentric cylinders. Of all, SWCNT exhibit the simplest geometry and have the lowest diameter ranging from $0.4 \mathrm{~nm}$ to $3 \mathrm{~nm}$ [2]. CNTs have been recently dispersed in a number of thermoset and thermoplastic polymers to obtain electrically conductive multifunctional composites with enhanced or retaining mechanical properties [3-9]. However, CNTs tend to form agglomerates during processing due to relatively strong van der Waals attractions between the individual tubes and their huge surface area, which makes it difficult to realize the desired enhancements in final properties of the resulting nanocomposites. From that point of view, homogeneous dispersion of CNTs within the corresponding matrix and the chemical interactions between nanotubes and polymer chains are the crucial subjects of interest to be considered prior to nanocomposite processing [7-9]. Therefore, their surfaces are chemically treated with a number of surface 
functional groups to improve their dispersion within the corresponding resin as well as interfacial adhesion between the CNT surface and the polymer matrix.

In some industrial applications such as exterior automotive parts, some textiles and household products static electric dissipation is expected from polymeric materials to avoid electrostatic charging [8-11]. For this purpose, conductive fillers such as carbon black (CB) are added to the corresponding polymers to obtain electrical conductivity above the required level of $\sigma=10^{-6} \mathrm{Sm}^{-1}$ [8]. However, the required $\mathrm{CB}$ content for polymers to accomplish the desired level of electrical conductivity is relatively high, such that the mechanical properties (fracture toughness and tensile strength) of the corresponding polymers is significantly reduced to some extent due to the high amount of the fillers [9-12]. On the other hand, application of highly conductive nanoparticles into an isolating polymer matrix is supposed to induce a higher electrical conductivity at a relatively low filler content when compared to conductive micro-particles.

Electrical conductivity is generally expressed by percolation theory. It implies that a conductive path way occurs once a critical filler concentration is achieved. Percolation threshold defines the transitions from insulator to conductor in composites and it is a strong function of the aspect ratio of the filler particles [13]. Therefore, it is more effective to employ CNTs with large aspect ratio instead of CB particles in spherical shapes to achieve a percolation threshold at comparatively lower filler contents. In the literature, reported amount of CNT loading within polymer matrices for a percolation threshold varies widely from less than $1 \%$ to over $10 \%$ in weight [14]. This relatively large range of the concentration is related with the parameters affecting the composite conductivities, such as aspect ratio and surface treatment of CNTs, orientation of nanotubes in the resin system, processing temperature and curing conditions [10]. In that manner, a number of theoretical percolation models have been proposed to identify the critical filler volume content, at which a conductive network is formed in polymers. In addition, temperature variation of conductivity is critical to investigate and transport mechanisms have been explained by various models, including Luttinger liquid, one dimensional disordered wire, activated process, variable range hopping and fluctuationinduced tunneling through the barriers between the metallic regions model [12-22].

The present study aims to investigate the electrical properties and its temperature dependence of polyesterbased nanocomposites containing various amounts of MWCNTs and DWCNTs with and without amine functional groups $\left(-\mathrm{NH}_{2}\right)$. Moreover, the effects of amine functional groups over surfaces of CNTs on the electrical behavior of nanocomposites will be discussed in detail.
Finally, temperature dependent electrical conductivities of CNT/polymer nanocomposites will be fitted to some analytical models to identify the mechanism involved for electrical conductivity.

\section{Materials}

Catalytically grown DWCNT and MWCNT with and without amine $\left(-\mathrm{NH}_{2}\right)$ functional groups were obtained from Nanocyl (Namur Belgium). Table 1 gives the specifications (aspect ratio, inner and outer diameter, length and density) of various types of CNTs, obtained from the producer. The density of the carbon nanotubes $\left(\rho_{\mathrm{CNT}}\right)$ given in the table was calculated based on the following equation suggested by Thostenson et al. [23],

$\rho_{\mathrm{CNT}}=\frac{\rho_{g}\left(d_{a}^{2}-d_{i}^{2}\right)}{d_{a}^{2}}$

In the equation, $d_{i}$ and $d_{a}$ are the inner and outer diameter of the nanotubes, respectively and $\rho_{g}$ is the density of graphite. Density of graphite was taken as $2.25 \mathrm{~g} /$ $\mathrm{cm}^{3}$ in the calculations.

The nanotubes described above were used as filler for the polyester resin blend in this study. The polyester resin was composed of Poliya 420 styrene free polyester, Poliya 240 commercial polyester and styrene, provided by Poliya Polyester Inc, Turkey. For adjustment of appropriate gelation time and viscosity during the real part processing, a polyester resin blend was formulated based on mixing of Poliya 420 (45 wt.\%), Poliya 240 (30 wt.\%) and styrene (25 wt.\%). Cobalt naphtanate (CoNAP) and methyl ethyl kethone peroxide (MEKP) were used as accelator and initiator, respectively to polymerize each corresponding polyester suspension.

\section{Experimental}

Preparation of nanocomposites

Poliya 420 (45 wt.\%) and Poliya 240 (30 wt.\%) were first mixed by hand in a cup for 10 min followed by addition of $0.1,0.3$ and 0.5 wt.\% of the CNTs into the prepared resin blends. To disperse the CNTs within the resin blend, a shear intensive mechanical blending (3-roll milling) technique was utilized. The dwell time of the suspension on the rolls was set about $2 \mathrm{~min}$. The CNT/polymer suspensions were collected by spatula and then $25 \mathrm{wt} . \%$ of styrene was introduced to each obtained suspension. Afterwards, 0.2 wt. $\%$ of CoNAP $(6 \%)$ and 1 wt. $\%$ of 
Table 1 Physical properties of carbon nanotubes (CNTs) used in this study [10]

\begin{tabular}{lclclc}
\hline Nanotube type & $\mathrm{L}($ length $)(\mu \mathrm{m})$ & $\mathrm{d}_{\text {in }}$ (inner diameter) $(\mathrm{nm})$ & $\mathrm{d}_{\text {out }}$ (outer diameter) $(\mathrm{nm})$ & $\rho_{\mathrm{CNT}}($ density $)\left(\mathrm{g} / \mathrm{cm}^{3}\right)$ & Aspect ratio \\
\hline DWCNT & 10 & 2.1 & 2.8 & 0.98 & 3570 \\
DWCNT-NH & 5 & 2.1 & 2.8 & 0.98 & 1785 \\
MWCNT & 50 & 4.0 & 15 & 2.09 & 3333 \\
MWCNT-NH & 10 & 4.0 & 15 & 2.09 & 666 \\
\hline
\end{tabular}

MEKP were introduced to the resin system, respectively, to polymerize the resin blends. The CNT/polyester suspensions were casted into aluminum molds and the samples were allowed to cure at room temperature followed by a post curing in an oven at $110^{\circ} \mathrm{C}$ for $2 \mathrm{~h}$.

Please note that finally the styrene was added separately to the corresponding resin system in order to avoid its evaporation during the 3 -roll milling processing due to heat evolved as a results of the intense shearing between the rolls. The evaporation of the styrene increases the viscosity of the resin left over the rolls, which causes reduction in the final properties of the nanocomposites.

\section{Measurements of electrical conductivity}

Temperature dependent electrical conductivity of composites was measured by conventional DC (four-point probe) method from room temperature down to $77 \mathrm{~K}$. The rectangular specimens were sectioned from larger samples. To check the homogeneity of the samples, electrical measurements were performed on various rectangular samples from the same batch. The four-point probe was contacted on samples with silver paint. Resistance versus temperature data of CNT/polymer nanocomposites was obtained using computer controlled data acquisition system.

\section{Results and discussion}

\section{Electrical conductivity}

Figure 1a shows the measured electrical conductivity of nanocomposites at room temperature with respect to volume content of CNTs. Note that the corresponding volume content of DWCNTs and MWCNTs were calculated based upon the densities given in Table 1 . The volume fraction of CNTs corresponding to weight percentages can be seen in Table 2. Figure 1a indicates that the presence of very low amount of CNTs within the polyester resin blend stimulated an electrical conduction in the resulting nanocomposites. Furthermore, an increasing CNT volume content lead to a rise in the conductivity of the nanocomposites with both MWCNT and DWCNTs. However, nanocomposites containing amino functionalized nanotubes $\left(\mathrm{CNT}-\mathrm{NH}_{2}\right)$ possessed lower electrical conductivity compared to those with untreated CNTs. As an example, electrical conductivities of nanocomposites containing 0.247 and 0.149 vol.\% of MWCNT-NH $\mathrm{NH}_{2}$ are six and hundred times lower than those of nanocomposites with untreated CNTs. On the other hand, the conductivity of the nanocomposites containing 0.105 vol. $\%$ of DWCNT- $\mathrm{NH}_{2}$ could not be measured because the value was not in the sensitivity range of the measurement device, while those of the nanocomposites with 0.316 and 0.523 vol. $\%$ of DWCNT-NH $\mathrm{DH}_{2}$ were easy to obtain. From a theoretical point of view, depending upon volume fraction, the carbon nanotubes with relatively high densities are anticipated to possess the lowest percolation threshold, which is very proportional to our findings as well. On the other hand, it was concluded that nanocomposites with the amino functionalized nanotubes have a higher percolation threshold than those with untreated nanotubes. These findings may be attributed to surface functionalization process. The amino groups are chemically attached onto the surfaces of CNTs via ball-milling process performed in ammonia solution by the manufacturer [10]. During the process, the CNTs are broken in length and their aspect ratios are reduced to some extent as shown in Table 1. As a result, the percolation threshold, which is particularly interrelated to the aspect ratio and volume content of the tubes, shifted to higher filler contents for nanocomposites containing amino functionalized nanotubes. Moreover, incorporation of amine funtional groups into a conjugated $\pi$-electron system as in the case of graphite structures like carbon nanotubes leads to formation of $\mathrm{sp} 3$ carbons, which carry the functional groups. Thus, the consequent modifications in structure interrupt the conjugation and induce a distortion of the graphitic layer. When handled in term of electron conduction, sp-3 carbons are very likely to be considered as defects, which reduce drastically the maximum conductivity of the individual nanotube. In brief, functionalization of the CNTs is presumed detrimental for the overall conductivity of their final polymer based products due to the formation of an isolating polymer layer around the nanotubes as a consequence of improved interactions between CNTs and the polymer, which causes higher contact resistance to occur between CNTs. In other words, enhanced chemical interactions at the interface between CNTs and polyester chains is very likely to occur 

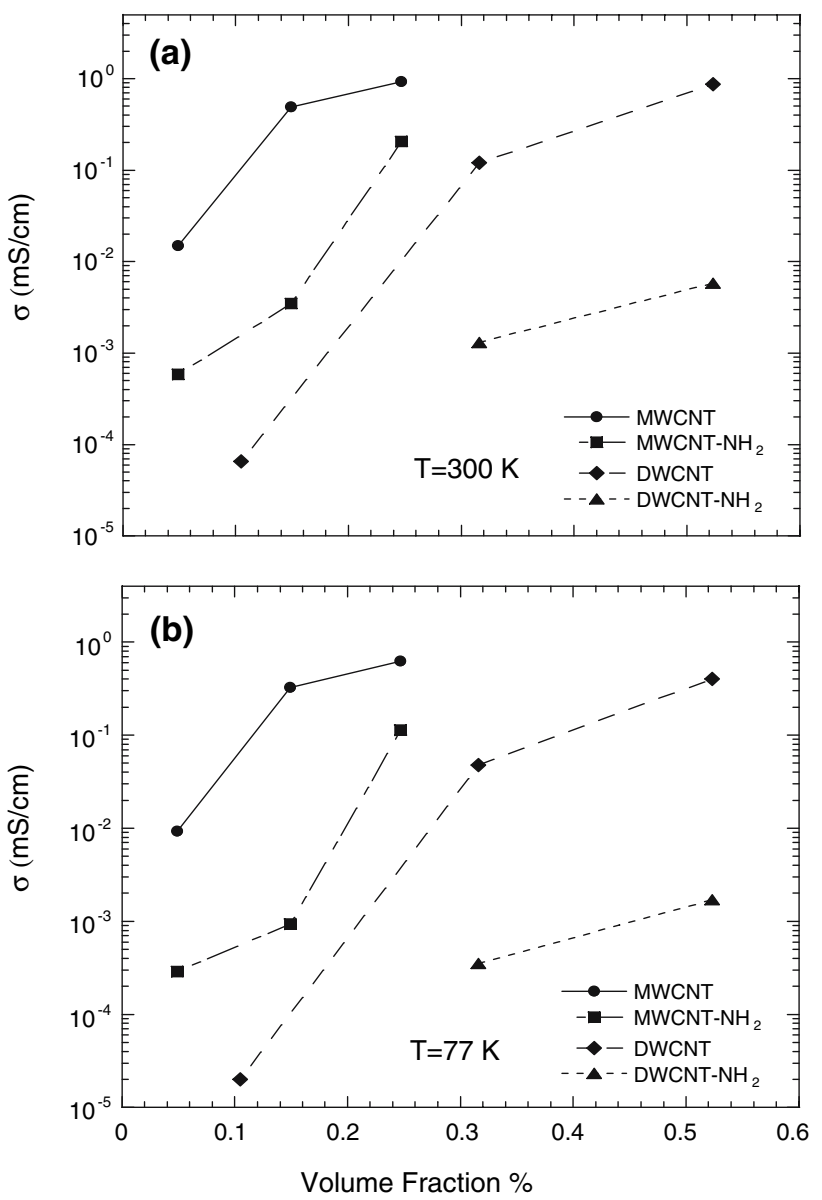

Fig. 1 Electrical conductivity of two different types of CNT polymer composites with and without amine functional groups, including MWCNT and DWCNT with different volume content. (a) room temperature, (b) $77 \mathrm{~K}$

Table 2 The volume fraction of CNTs corresponding to weight percentages

\begin{tabular}{lll}
\hline Weight\% & $\begin{array}{l}\text { Volume\% }(\mathrm{DWCNT}) \text { or } \\
\left(\mathrm{DWCNT}-\mathrm{NH}_{2}\right)\end{array}$ & $\begin{array}{l}\text { Volume\% (MWCNT) or } \\
\left(\mathrm{MWCNT}-\mathrm{NH}_{2}\right)\end{array}$ \\
\hline 0.1 & 0.105 & 0.049 \\
0.3 & 0.316 & 0.149 \\
0.5 & 0.523 & 0.247 \\
\hline
\end{tabular}

with some chemical reactions between the polyester chains and amine groups over CNT surfaces, which raises the resistivity of the corresponding nanocomposites. However, it is a big challenge to monitor these complex reactions, including the interactions between styrene, polyester resins and amine functional groups due to complicated free radical polymerization of the corresponding resin systems. The determination of these reactions and interactions are not the subject of the present paper and they will be addressed in the further publications.
The results indicated that the variations in conductivity of nanocomposites containing DWCNTs are relatively larger than those with MWCNTs. This implies that DWCNTs need to be added into polyester resin blend with relatively higher concentrations to achieve the same electrical conductivity of the nanocomposites containing MWCNTs. A relatively higher tendency of DWCNTs for agglomeration within the corresponding resin system due to their so pronounced large surface area may associate with higher resistivity of the nanocomposites with DWCNTs as compared to those with MWCNTs.

The electrical conductivity of the nanocomposites at $77 \mathrm{~K}$ is illustrated in Fig. 1b. It was found that the conductivity of the samples at $77 \mathrm{~K}$ is 1.5 to 3.5 times lower than those of the samples at room temperature.

Figure 2 shows normalized resistivity versus temperature curves of nanocomposites with $0.3 \mathrm{wt} . \%$ MWCNT. The data shown in the figure were taken from two different samples to evaluate the homogeneity of the composites. The samples exhibited a conductivity of 0.49 and $0.44 \mathrm{mS} /$ $\mathrm{cm}$ at room temperature. As seen in the figure, both of the samples showed the same temperature dependence, as it is expected. A similar behavior was obtained for all the nanocomposites prepared with various CNT contents as well. So, the presented resistivity curves are representative material properties. On the other hand, the maximum deviation of resistivity values was found to be $75 \%$ in nanocomposites containing $0.3 \mathrm{wt} . \%$ of DWCNT-NH relatively large deviation of the resistivity for this group of samples might be related with the reduced aspect ratios and higher agglomeration tendency of the DWCNT- $\mathrm{NH}_{2}$ in the matrix, as discussed in detail previously.

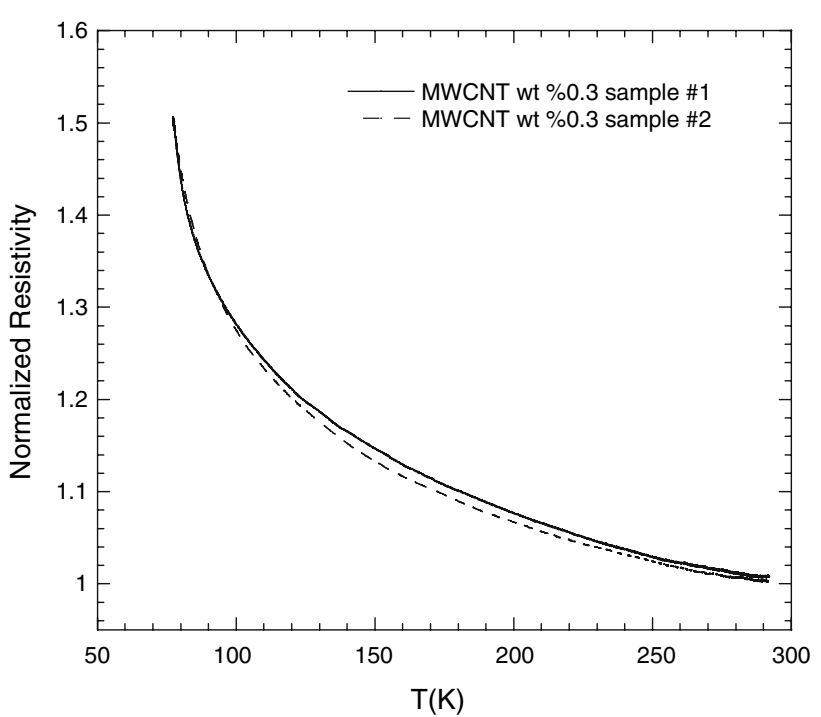

Fig. 2 Normalized resistivity versus temperature of $0.3 \mathrm{wt} . \%$ MWCNT from two distinct samples 
Figure 3 shows the normalized resistivity versus temperature for MWCNT and DWCNT nanocomposites at various CNT contents with and without amine functional groups for comparison. The results of temperature dependence of resistivity showed that nanocomposites exhibited semiconductor like behavior regardless of the types of carbon nanotubes (MWCNT or DWCNT) or the presence of functional groups over the CNTs. On the other hand, the resistivity values rapidly increase below $100 \mathrm{~K}$. A similar finding was reported in the literature [15] that nanocomposites exhibit a valley in temperature dependence data. It was explained that the metallic conduction is a dominant mechanism around room temperature while at low temperatures tunneling becomes the foremost conduction mechanism. In our samples, we obtain only increasing resistivity with decreasing temperature, so tunneling is likely the dominant mechanism.

\section{Tunneling fit}

The electrical conductivity mechanisms in CNT/polymer nanocomposites were investigated using various models in the literature; such as Luttinger liquid model [16], one dimensional disordered wire model [17], activated process model [18], variable range hopping model [19] and fluctuation induced tunneling through the barriers between the metallic regions model $[15,20]$. Of all these models, the fluctuation induced tunneling model (FITM) based on thermally activated voltage fluctuations across insulating gaps in disordered materials such as CNT/polymer nanocomposites was found to match well with our temperature dependent experimental data. In our nanocomposites, there are large conduction regions, i.e.; CNTs separated by small insulating barriers (polyester resin). The thickness and height of the tunneling barrier plays an important role in determining the temperature and magnetic field dependence of conductivity since electrons tunnel through insulator regions. In other words, the electron tunneling probability exponentially depends on the insulating barrier thickness changing with temperature. Also, it is known that the degree of nonlinearity of tunneling current decreases as the temperature increases, due to narrowing of barrier by fluctuation. According to FITM the temperature dependence of electrical resistivity, $\rho$, is given by the equation below [15];

$\rho=\rho_{0} \exp \left(\frac{T_{t}}{T_{s}+T}\right)$

where $\rho_{o}$ is the resistivity at $T \gg T_{t}$ and $T_{s}$. In other words, at very high temperatures the resistivity is independent of temperature and it approaches to $\rho_{o}$. In the equation, $T_{t}$ is a measure of the energy required to move an electron across
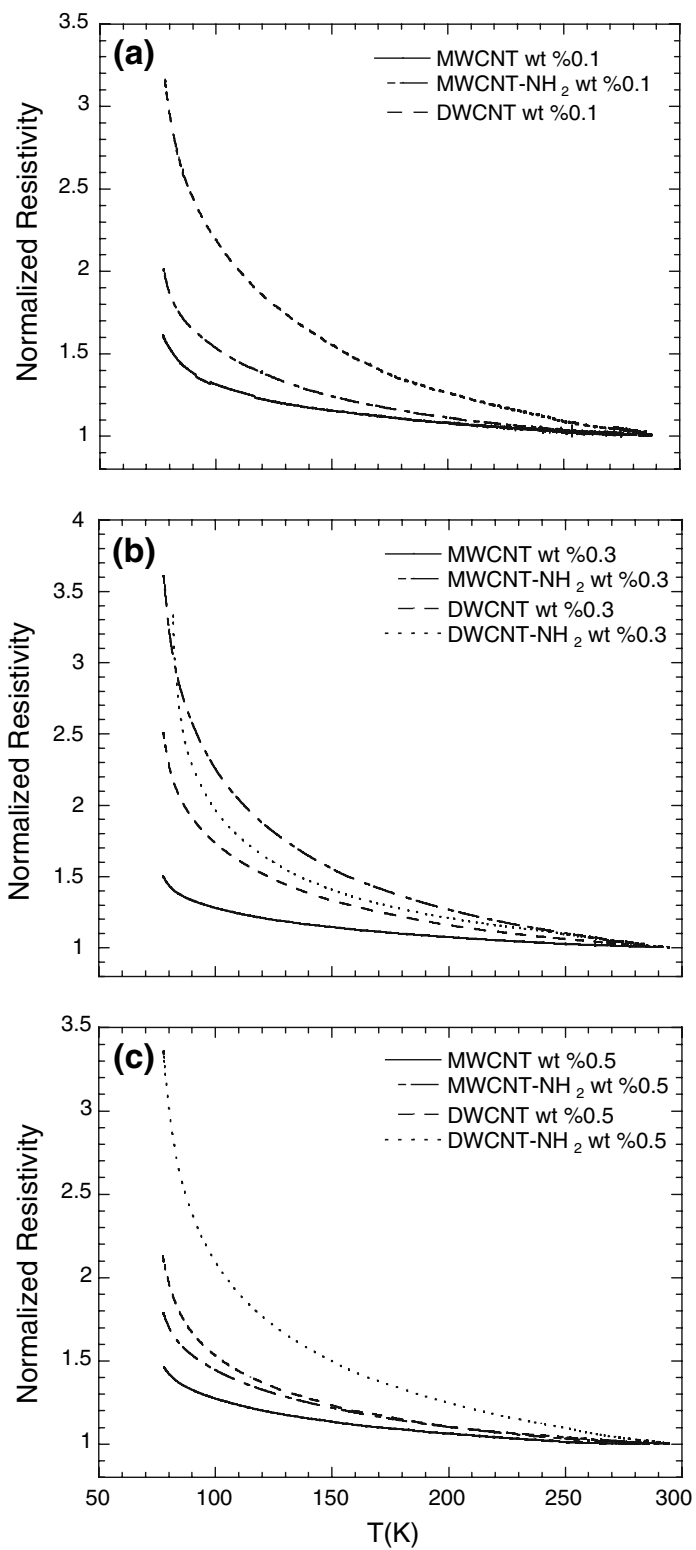

Fig. 3 Normalized resistivity versus temperature of CNT polymer nanocomposites with various weight fractions (a) $0.1 \mathrm{wt} . \% \mathrm{CNTs}$, (b) 0.3 wt. $\%$ CNTs, (c) 0.5 wt. $\%$ CNTs

the insulating gap where the magnitude of the thermal fluctuations become comparable to the magnitude of the tunneling barriers. $T_{s}$ is a temperature much below which the resistivity reduces to temperature independent tunneling [20, 21]. Detailed explanations of $T_{t}$ and $T_{s}$ in terms of various parameters such as height of the potential barrier, size of the barrier can be found in [24].

Figure $4 \mathrm{a}, \mathrm{b}$ show the experimental results of temperature dependence of resistivity for nanocomposites prepared with DWCNT at 0.1 and 0.3 wt.\% loading in comparison with FITM predictions. The parameters $T_{t}$ and $T_{s}$ were found to be 132 and $8 \mathrm{~K}$ for nanocomposites with $0.1 \mathrm{wt} . \%$ 
of DWCNT and 91 and $2 \mathrm{~K}$ for nanocomposites with 0.3 wt.\% of DWCNT. As seen in the figures, the FITM model fits well with the experimental data obtained at high temperatures; however, below $100 \mathrm{~K}$ a deviation becomes more visible from the fitting curve. A similar behavior was reported in the literature recently for single walled carbon nanotubes (SWCNTs)/polymer composites [22]. Based on the analysis of Fig. 4 using FITM, temperature dependence of resistivity shows that above $T_{s}=8 \mathrm{~K}$ thermally activated conduction over the barrier begin to occur. In addition, the change in resistivity above the temperature $T_{t}=132 \mathrm{~K}$ begin to decrease because of reducing domination of charge carrier tunneling through the barrier. Above this temperature, electron-electron and electronphonon interactions begin to occur and contribute to resistivity. In general, resistivities predicted by these fit parameters correlate well with our experimental results at temperatures between $100 \mathrm{~K}$ and $300 \mathrm{~K}$. However, they are not explicit values to form general aspect with respect to different filler content and attachment of amine functional groups over the surface.
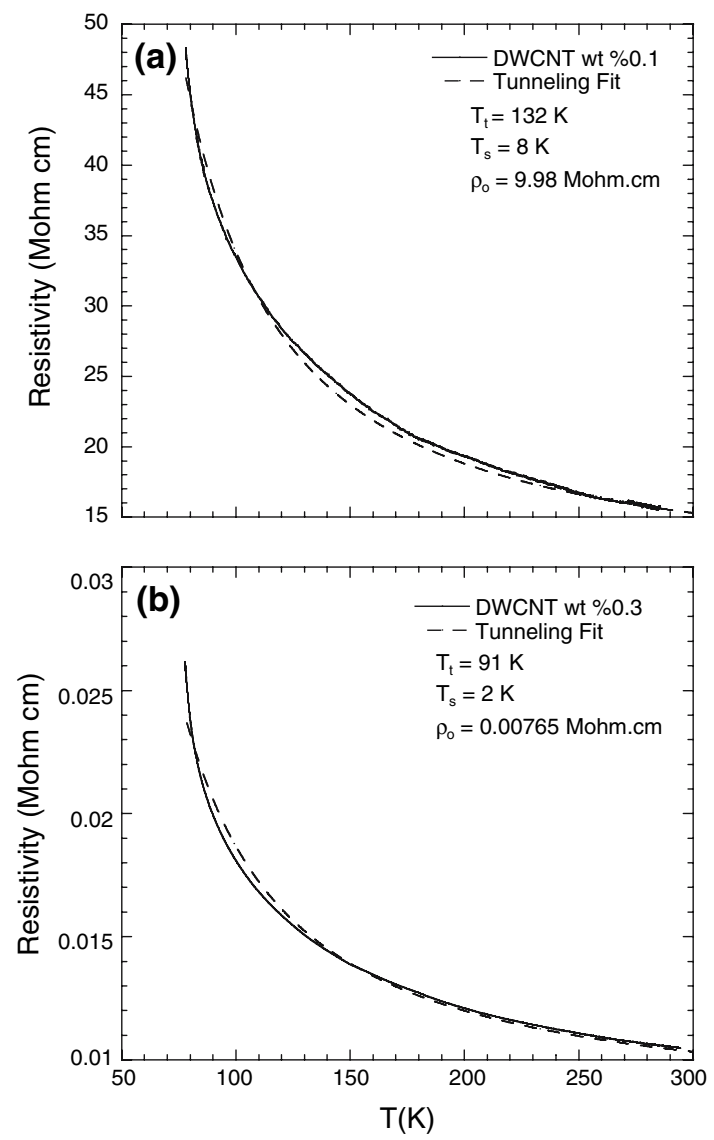

Fig. 4 Temperature dependence of resistivity (solid lines) and FITM model fits (dashed lines) for (a) $0.1 \mathrm{wt} \%$ DWCNT, (b) $0.3 \mathrm{wt} \%$ DWCNT. The fits follow experimental data for high temperatures and there is a strong deviation at low temperatures
Figures 5a, b show the temperature dependence of resistivity for nanocomposites containing $0.3 \mathrm{wt} \%$ of MWCNT with and without amine functional groups. The dashed lines represent FITM fits. The presence of aminefunctionalized nanotubes in the resin system increases $T_{t}$ from $42 \mathrm{~K}$ to $152 \mathrm{~K}$, in which the tunnel barrier is increasing between the CNTs. Since CNTs with amine functional groups have higher $T_{t}$ values than those without amine functional groups, the conduction is dominated by tunneling of charge carriers for nanocomposites with amine functional CNTs at room temperature.

\section{Conclusion}

CNT/polyester nanocomposites were successfully produced by using 3-roll mill process technique and their temperature dependence of electrical conductivity were measured performing the four probe test method. It was found that the incorporation of very low amount of CNTs into the resin system induced an electrical conduction in the resulting nanocomposites. It was also found that
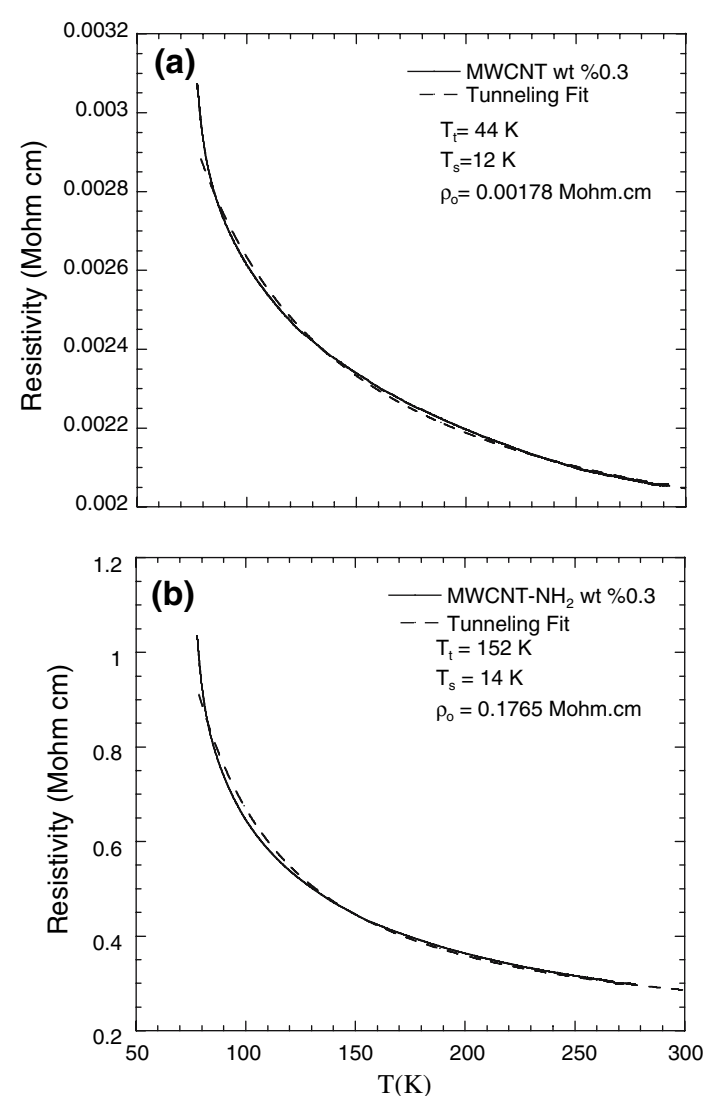

Fig. 5 Temperature dependence of resistivity (solid lines) and FITM model fits (dashed lines) for (a) 0.3 wt.\% MWCNT, (b) 0.3 wt.\% MWCNT-NH ${ }_{2} . T_{t}$ is considerably larger for functionalized CNT loadings 
increasing the carbon nanotubes filler content within insulating polymer matrices yields electrically percolating networks and electrical conductivity increased with increasing content of nanotubes in the matrix.

Nanocomposites containing CNTs with amine functional groups exhibited lower electrical conductivity as compared to those with untreated ones. These findings were attributed to surface functionalization process in which the amino groups are chemically attached onto the surfaces of CNTs. During the functionalization process, the aspect ratio of the CNTs is reduced and the distortion of the graphitic structure, which leads to the reduced conductivity, is expected to occur. Moreover, the amine groups may react with the polyester chains and form an electrically insulating layer around the tubes, which may act as a barrier between individual tubes and increases the resistivity of the nanocomposites.

Electrical conductivity of nanocomposites with MWCNT was obtained to be higher than those with DWCNTs at the same filler content due to the relatively higher tendency of DWCNTs for agglomeration within the corresponding resin system.

The results of temperature dependence of resistivity showed that nanocomposites exhibited semiconductor like behavior regardless of the types of carbon nanotubes (MWCNT or DWCNT) or the presence of functional groups over the CNTs. Also, we obtain an increasing resistivity with decreasing temperature, so tunneling is likely the dominant mechanism.

It was observed that the results predicted by the fluctuation induced tunneling model were in very good agreement with those experimentally obtained temperature dependence of resistivity. The parameters $T_{t}, T_{s}$ and $\rho_{0}$ were found for each CNT/polymer nanocomposites based on the model.

Acknowledgement Authors acknowledge the financial support from TÜBITAK-JÜLICH 5 Project. Also, L.O. acknowledges support from the Turkish Academy of Sciences, in the framework of the Young Scientist Award Program (LO/TUBA-GEBIP/2002-1-17).

\section{References}

1. Iijima S (1991) Nature (London) 354:56

2. Balasubramanian K, Burghard M (2005) Small 1(2):180

3. Khare R, Bose S (2005) J Min Mater Character Eng 4:31

4. Yu MF, Lourie O, Dyer MJ, Moloni K, Kelly TF, Ruoff RS (2000) Science 287:637

5. Allaoui A, Bai S, Cheng HM, Bai JB (2002) Compos Sci Technol 62:1993

6. Kim P, Shi L, Majumdar A, Maceuen PL (2001) Phys Rev Lett 87:5502

7. Martin CA, Sandler JKW, Windle AH, Schwarz MK, Bauhofer W, Schulte K, Shaffer MSP (2005) Polymer 46:877

8. Sandler J, Schaffer MSP, Prasse T, Bauhofer W, Schulte K, Windle AH (1999) Polymer 40:5967

9. Du F, Scogna RC, Zhou W, Brand S, Fischer JE, Winey KI (2004) Macromolecules 37:9048

10. Gojny FH, Wichmann MHG, Fiedler B, Kinloch IA, Bauhofer W, Windle AH, Schulte K (2006) Polymer 47:2036

11. Kymakis E, Alexandaou I, Amaratunga GAJ (2002) Synthetic Met 127:59

12. Astorga HR, Mendoza D (2005) Opt Mater 27:1228

13. Sandler JKW, Kirk JE, Kinloch IA, Schaffer MSP, Windle AH (2003) Polymer 44:893

14. Jiang X, Bin Y, Matsuo M (2005) Polymer 46:7418

15. Sheng P (1980) Phys Rev B 21:2180

16. Bockrath M, Cobden DH, Lu J, Rinzler AG, Smalley RE, Balemts L, Mcquen PL (1999) Nature 397:598-601

17. Mischenko EG, Andreev AV, Glazman LI (2001) Phys Rev Lett $87: 246801$

18. Meyorsan B, Smith FW (1980) Solid State Commun 34:531

19. Mott NF, Davis EA (1979) In: Electronic processes in NonCrystalline Materials Clarendon, Oxford, p 231

20. Kim GT, Jhang SH, Park JG, Park YW, Roth S (2001) Synthetic Met 117:123

21. Sichel EK, Sheng P, Gittlemann JI, Bozowski S (1981) Phys Rev B 24:6131

22. Kymakis E, Amaratunga GAJ (2006) J Appl Phys 99:084302

23. Thostenson ET, Chouj TW (2003) Phys D: Appl Phys 36:573582

24. Bajpai A, Nigam AK (2007) Phys Rev B 75:064403 